International Journal of Difference Equations

ISSN 0973-6069, Volume 14, Number 1, pp. 39-58 (2019)

https://dx.doi.org/10.37622/IJDE/14.1.2019.39-58

\title{
Improved Oscillation Tests for Retarded Difference Equations
}

\author{
George E. Chatzarakis \\ School of Pedagogical and Technological Education (ASPETE) \\ Department of Electrical and Electronic Engineering Educators \\ Athens, 14121, Greece
}

\author{
Irena Jadlovská \\ Technical University of Košice \\ Department of Mathematics and Theoretical Informatics \\ Košice, 04200, Slovakia
}

\begin{abstract}
In this paper, some new oscillation criteria for retarded difference equations with nonnegative coefficients and several arguments are established, based on an iterative procedure. An example illustrating the applicability and strength of the results, numerically solved in MATLAB, is also given.
\end{abstract}

AMS Subject Classifications: 39A10, 39A21.

Keywords: Difference equation, nonmonotone arguments, oscillatory solutions, nonoscillatory solutions.

\section{Introduction}

The rapid evolution of applied science and technology, especially in the domain of computer science, control systems and communications have contributed to researchers from the science of mathematics more necessary than ever. It is because of the continuous need to develop proper software and hardware, which primarily implies the construction of components that at first make possible the digitalization of data to transfer and then recover back again in the analogue form, that man is familiar with and understand.

Received January 12, 2019; Accepted August 1, 2019

Communicated by Mehmet Ünal 
However, these needs are directly related to the problematic scientific field of difference equations, since such equations describe all the components of the above mentioned technologies. This happens because such components always simulate an estimation relation to linear and time invariable systems where the next signal value is calculated based on many previous signal values and the very previous one. These recursive relations are described by recursive equations known as retarded difference equations.

Besides the purely mathematical problem, the interest in the behavior of the solutions to difference equations with retarded arguments is justified by the fact that the mathematical modeling of many real-world problems leads to difference equations where the unknown function depends on the past history rather than only the present state. This interest grows stronger as difference equations naturally arise from the discretization of differential equations.

Thus, all of the above make the theory of retarded difference equations very attractive to researchers. As a result, in the last decades many of them have focused their interest on problems of this area.

One of the most interesting problems is the study of the oscillation of solutions of retarded difference equations because it is related with the solution oscillations around an equilibrium point, regardless of their asymptotic behavior. See, for example, [1-20] and the references cited therein. However, the possibility of estimating such behaviors is especially important to the design of appliances and components used extensively in modern technologies, e.g. in the design of digital filters widely used in telecommunications.

A great part of the existing literature on the oscillation of retarded difference equations concerns the case where the arguments are nondecreasing, while only a small number of papers are dealing with the general case of arguments being not necessarily monotone. See, for example, [2-6,9] and the references cited therein. The consideration of nonmonotone arguments may lead to better approximation of the natural phenomena described by difference equations because quite often there appear natural disturbances such as noise in communication systems that affect all the parameters of the equation and therefore the "fair" (from a mathematical point of view) monotone arguments are, in fact, nonmonotone almost always. In view of this, an interesting question is whether we can state oscillation criteria considering the arguments to be not necessarily monotone.

Consider the difference equation with several retarded arguments of the form

$$
\Delta x(n)+\sum_{i=1}^{m} p_{i}(n) x\left(\tau_{i}(n)\right)=0 \quad n \in \mathbb{N}_{0},
$$

where, $\Delta$ denotes the forward difference operator $\Delta x(n)=x(n+1)-x(n)$.

Equation $(E)$ is studied under the following assumptions: everywhere $\left(p_{i}(n)\right)_{n \geq 0}$, $1 \leq i \leq m$, are sequences of nonnegative real numbers and $\left(\tau_{i}(n)\right)_{n \geq 0}, 1 \leq i \leq m$, are 
sequences of integers such that

$$
\tau_{i}(n) \leq n-1, \quad \forall n \in \mathbb{N}_{0} \quad \text { and } \quad \lim _{n \rightarrow \infty} \tau_{i}(n)=\infty, \quad 1 \leq i \leq m .
$$

Set

$$
v=-\min _{\substack{n \geq 0 \\ 1 \leq i \leq m}} \tau_{i}(n) .
$$

Clearly, $v$ is a finite positive integer, if (1.1) holds.

By a solution of $(E)$, we mean a sequence of real numbers $(x(n))_{n \geq-v}$ which satisfies $(E)$, for all $n \geq 0$. It is clear that, for each choice of real numbers $c_{-v}, c_{-v+1}, \ldots$, $c_{-1}, c_{0}$, there exists a unique solution $(x(n))_{n \geq-v}$ of $(E)$ which satisfies the initial conditions $x(-v)=c_{-v}, x(-v+1)=c_{-v+1}, \ldots, x(-1)=c_{-1}, x(0)=c_{0}$. When the initial data is given, we can obtain a unique solution to $(E)$ by using the method of steps.

A solution $(x(n))_{n \geq-v}$ of $(E)$ is called oscillatory, if the terms $x(n)$ of the sequence are neither eventually positive nor eventually negative. Otherwise, the solution is said to be nonoscillatory.

Throughout this paper, we are going to use the following notation:

$$
\begin{gathered}
\sum_{i=k}^{k-1} A(i)=0 \quad \text { and } \prod_{i=k}^{k-1} A(i)=1 \\
\alpha:=\liminf _{n \rightarrow \infty} \sum_{i=1}^{m} \sum_{j=\tau(n)}^{n-1} p_{i}(j)
\end{gathered}
$$

and

$$
D(\omega):= \begin{cases}0, & \text { if } \omega>1 / e \\ \frac{1-\omega-\sqrt{1-2 \omega-\omega^{2}}}{2}, & \text { if } \omega \in[0,1 / e]\end{cases}
$$

where $\tau(n)=\max _{1 \leq i \leq m} \tau_{i}(n)$ and $\tau_{i}(n), 1 \leq i \leq m$, are nondecreasing.

In 2006, Berezansky and Braverman [1] and in 2014, Chatzarakis, Pinelas and Stavroulakis [10] proved that if

$$
\limsup _{n \rightarrow \infty} \sum_{i=1}^{m} p_{i}(n)>0 \quad \text { and } \quad \alpha>\frac{1}{e}
$$

or

$$
\limsup _{n \rightarrow \infty} \sum_{i=1}^{m} \sum_{j=\tau(n)}^{n} p_{i}(j)>1
$$

respectively, then all solutions of $(E)$ are oscillatory.

Assume that the arguments $\tau_{i}(n), 1 \leq i \leq m$ are not necessarily monotone. 
Set

$$
h(n)=\max _{1 \leq i \leq m} h_{i}(n) \quad \text { where } \quad h_{i}(n)=\max _{0 \leq s \leq n} \tau_{i}(s), \quad n \geq 0
$$

and

$$
\begin{gathered}
a_{1}(n, k):=\prod_{i=k}^{n-1}\left[1-\sum_{\ell=1}^{m} p_{\ell}(i)\right] \\
a_{r+1}(n, k):=\prod_{i=k}^{n-1}\left[1-\sum_{\ell=1}^{m} p_{\ell}(i) a_{r}^{-1}\left(i, \tau_{\ell}(i)\right)\right], \quad r \in \mathbb{N} .
\end{gathered}
$$

Clearly, $h_{i}(n), h(n)$ are nondecreasing and $\tau_{i}(n) \leq h_{i}(n) \leq h(n) \leq n-1$, for all $n \geq 0$.

In 2015, Braverman, Chatzarakis and Stavroulakis [2] proved that if there exists a subsequence $\theta(n), n \in \mathbb{N}$ of positive integers such that

$$
\sum_{i=1}^{m} p_{i}(\theta(n)) \geq 1, \quad \forall n \in \mathbb{N},
$$

then all solutions of $(E)$ are oscillatory.

Under the assumption that

$$
\sum_{i=1}^{m} p_{i}(n)<1, \quad \forall n \geq 0,
$$

the same authors proved that if for some $r \in \mathbb{N}$

$$
\limsup _{n \rightarrow \infty} \sum_{j=h(n)}^{n} \sum_{i=1}^{m} p_{i}(j) a_{r}^{-1}\left(h(n), \tau_{i}(j)\right)>1,
$$

then all solutions of $(E)$ are oscillatory.

In 2018, Chatzarakis, Horvat-Dmitrović and Pašić [3] proved that, if for some $\ell \in \mathbb{N}$

$$
\limsup _{n \rightarrow \infty} \sum_{j=h(n)}^{n} P(j) \prod_{i=\tau(j)}^{h(n)-1} \frac{1}{1-\mathcal{P}_{\ell}(i)}>1
$$

or

$$
\limsup _{n \rightarrow \infty} \sum_{j=h(n)}^{n} P(j) \prod_{i=\tau(j)}^{h(n)-1} \frac{1}{1-\mathcal{P}_{\ell}(i)}>1-D(\alpha)
$$

where

$$
\mathcal{P}_{\ell}(n)=P(n)\left[1+\sum_{i=\tau(n)}^{n-1} \mathcal{P}(i) \prod_{j=\tau(i)}^{h(n)-1} \frac{1}{1-\mathcal{P}_{\ell-1}(j)}\right]
$$


with $\mathcal{P}_{0}(n)=P(n)=\sum_{i=1}^{m} p_{i}(n)$, then all solutions of $(E)$ are oscillatory.

Several improvements were made to the above conditions, see [4-6,9] to arrive at the recent forms [6]

$$
\begin{aligned}
\limsup _{n \rightarrow \infty} \sum_{\ell=h(n)}^{n} P(\ell) \exp \left(\sum_{j=\tau(\ell)}^{h(n)-1} P(j) \prod_{u=\tau(j)}^{j-1} \frac{1}{1-Z_{w}(u)}\right)>1, \\
\limsup _{n \rightarrow \infty} \sum_{\ell=h(n)}^{n} P(\ell) \exp \left(\sum_{j=\tau(\ell)}^{h(n)-1} P(j) \prod_{u=\tau(j)}^{j-1} \frac{1}{1-Z_{w}(u)}\right)>1-D(\alpha), \\
\limsup _{n \rightarrow \infty} \sum_{\ell=h(n)}^{n} \bar{P}(\ell) \exp \left(\sum_{j=\tau(\ell)}^{n} \bar{P}(j) \prod_{u=\tau(j)}^{j-1} \frac{1}{1-Z_{w}(u, \epsilon)}\right)>\frac{1}{D(\alpha)}-1,
\end{aligned}
$$

where

$$
Z_{w}(n)=P(n)\left[1+\sum_{i=\tau(n)}^{n-1} P(i) \exp \left(\sum_{j=\tau(i)}^{n-1} P(j) \prod_{u=\tau(j)}^{j-1} \frac{1}{1-Z_{w-1}(u)}\right)\right]
$$

with $Z_{0}(n)=P(n)\left[1+\sum_{i=\tau(n)}^{n-1} P(i) \exp \left(\lambda_{0} \sum_{j=\tau(i)}^{n-1} P(j)\right)\right]$,

where $P(n)=\sum_{i=1}^{m} p_{i}(n)$ and $\lambda_{0}$ is the smaller root of the transcendental equation $\lambda=e^{\alpha \lambda}$.

\section{Main Results}

We further study $(E)$ and derive new sufficient oscillation conditions, involving lim sup, which improve on all previously known results, in the literature. The proofs of the main results are essentially based on the following lemmas.

Lemma 2.1 (See [5, Lemma 1]). Assume that $h(n)$ is defined by (1.4) and $\alpha>0$. Then

$$
\liminf _{n \rightarrow \infty} \sum_{i=1}^{m} \sum_{j=h(n)}^{n-1} p_{i}(j)=\liminf _{n \rightarrow \infty} \sum_{i=1}^{m} \sum_{j=\tau(n)}^{n-1} p_{i}(j)=\alpha .
$$

Lemma 2.2 (See [5, Lemma 1]). Assume that $h(n)$ is defined by (1.4), $0<\alpha \leq 1 / e$ and $x(n)$ is an eventually positive solution of $(E)$. Then

$$
\liminf _{n \rightarrow \infty} \frac{x(h(n))}{x(n)} \geq \lambda_{0}
$$

where $\lambda_{0}$ is the smaller root of the transcendental equation $\lambda=e^{\alpha \lambda}$. 
The next lemma provides a lower estimate for the ratio $x(n+1) / x(h(n))$ in terms of the smaller root of $d^{2}-(1-\alpha) d+\alpha^{2} / 2=0$. The proof of this lemma is similar to the proof of [11, Lemma 2.1].

Lemma 2.3. Assume that $h(n)$ is defined by (1.4), $0<\alpha \leq 1 / e$ and $x(n)$ is an eventually positive solution of $(E)$. Then

$$
\liminf _{n \rightarrow \infty} \frac{x(n+1)}{x(h(n))} \geq D(\alpha) .
$$

Theorem 2.4. Assume that $h(n)$ is defined by (1.4). If for some $\xi \in \mathbb{N}$

$$
\limsup _{n \rightarrow \infty} \sum_{k=h(n)}^{n} P(k) \exp \left(\sum_{\ell=\tau(k)}^{h(n)-1} P(\ell) \exp \left(\sum_{j=\tau(\ell)}^{\ell-1} P(j) \prod_{u=\tau(j)}^{j-1} \frac{1}{1-D_{\xi}(u)}\right)\right)>1,
$$

where

$$
\begin{aligned}
& D_{\xi}(n)=P(n) \times \\
& \times\left[1+\sum_{k=\tau(n)}^{n-1} P(k) \exp \left(\sum_{\ell=\tau(k)}^{n-1} P(\ell) \exp \left(\sum_{j=\tau(\ell)}^{\ell-1} P(j) \prod_{u=\tau(j)}^{j-1} \frac{1}{1-D_{\xi-1}(u)}\right)\right]\right.
\end{aligned}
$$

with

$$
D_{0}(n)=P(n)\left[1+\sum_{k=\tau(n)}^{n-1} P(k) \exp \left(\sum_{\ell=\tau(k)}^{n-1} P(\ell) \exp \left(\lambda_{0} \sum_{j=\tau(\ell)}^{\ell-1} P(j)\right)\right]\right.
$$

and $\lambda_{0}$ is the smaller root of the transcendental equation $\lambda=e^{\alpha \lambda}$, then all solutions of (E) are oscillatory.

Proof. Assume, for the sake of contradiction, that there exists a nonoscillatory solution $(x(n))_{n \geq-v}$ of $(E)$. Since $(-x(n))_{n \geq-v}$ is also a solution of $(E)$, we can confine our discussion only to the case where $x(n)>0$, for all large $n$. Let $n_{1} \geq-v$ be an integer such that $x(n)>0$, for all $n \geq n_{1}$. Then, there exists $n_{2} \geq n_{1}$ such that $x\left(\tau_{i}(n)\right)>0$, for all $n \geq n_{2}$. In view of this, $(E)$ becomes

$$
\Delta x(n)=-\sum_{i=1}^{m} p_{i}(n) x\left(\tau_{i}(n)\right) \leq 0, \text { for all } n \geq n_{2},
$$

which means that $(x(n))$ is an eventually nonincreasing sequence of positive numbers. Dividing $(E)$ by $x(n)$ and summing up from $\tau(n)$ to $n-1$, we take

$$
\sum_{j=\tau(n)}^{n-1} \frac{\Delta x(j)}{x(j)}=-\sum_{j=\tau(n)}^{n-1} \sum_{i=1}^{m} p_{i}(j) \frac{x\left(\tau_{i}(j)\right)}{x(j)} \leq-\sum_{j=\tau(n)}^{n-1}\left(\sum_{i=1}^{m} p_{i}(j)\right) \frac{x(\tau(j))}{x(j)}
$$


or

$$
\sum_{j=\tau(n)}^{n-1} \frac{\Delta x(j)}{x(j)} \leq-\sum_{j=\tau(n)}^{n-1} P(j) \frac{x(\tau(j))}{x(j)}
$$

where $P(n)=\sum_{i=1}^{m} p_{i}(n)$.

However, since $e^{x} \geq x+1, x \in \mathbb{R}$ we have

$$
\begin{aligned}
\sum_{j=\tau(n)}^{n-1} \frac{\Delta x(j)}{x(j)} & =\sum_{j=\tau(n)}^{n-1}\left(\frac{x(j+1)}{x(j)}-1\right) \\
& =\sum_{j=\tau(n)}^{n-1}\left[\exp \left(\ln \frac{x(j+1)}{x(j)}\right)-1\right] \\
& \geq \sum_{j=\tau(n)}^{n-1} \ln \frac{x(j+1)}{x(j)}=\ln \frac{x(n)}{x(\tau(n))}
\end{aligned}
$$

Combining $(E),(2.6)$ and (2.7), we obtain

$$
\Delta x(n)+p(n) x(n) \exp \left(\sum_{j=\tau(n)}^{n-1} P(j) \frac{x(\tau(j))}{x(j)}\right) \leq 0 .
$$

Dividing (2.8) by $x(n)$ and summing up from $\tau(k)$ to $n-1$, we take

$$
\sum_{\ell=\tau(k)}^{n-1} \frac{\Delta x(j)}{x(j)} \leq-\sum_{\ell=\tau(k)}^{n-1} P(\ell) \exp \left(\sum_{j=\tau(\ell)}^{\ell-1} P(j) \frac{x(\tau(j))}{x(j)}\right)
$$

which, in view of (2.7), gives

$$
\ln \frac{x(n)}{x(\tau(k))} \leq-\sum_{\ell=\tau(k)}^{n-1} P(\ell) \exp \left(\sum_{j=\tau(\ell)}^{\ell-1} P(j) \frac{x(\tau(j))}{x(j)}\right)
$$

or

$$
x(\tau(k)) \geq x(n) \exp \left(\sum_{\ell=\tau(k)}^{n-1} P(\ell) \exp \left(\sum_{j=\tau(\ell)}^{\ell-1} P(j) \frac{x(\tau(j))}{x(j)}\right)\right) .
$$

Summing up (E) from $\tau(n)$ to $n-1$, we have

$$
0=x(n)-x(\tau(n))+\sum_{k=\tau(n)}^{n-1} \sum_{i=1}^{m} p_{i}(k) x\left(\tau_{i}(k)\right)
$$




$$
\begin{aligned}
& \geq x(n)-x(\tau(n))+\sum_{k=\tau(n)}^{n-1}\left(\sum_{i=1}^{m} p_{i}(k)\right) x(\tau(k)) \\
& =x(n)-x(\tau(n))+\sum_{k=\tau(n)}^{n-1} P(k) x(\tau(k)) .
\end{aligned}
$$

Combining (2.9) and (2.10), we obtain

$$
x(n)-x(\tau(n))+x(n) \sum_{k=\tau(n)}^{n-1} P(k) \exp \left(\sum_{\ell=\tau(k)}^{n-1} P(\ell) \exp \left(\sum_{j=\tau(\ell)}^{\ell-1} P(j) \frac{x(\tau(j))}{x(j)}\right)\right) \leq 0 .
$$

Multiplying the last inequality by $P(n)$, we get

$$
\begin{aligned}
& x(n) P(n)-P(n) x(\tau(n)) \\
& +P(n) x(n) \sum_{k=\tau(n)}^{n-1} P(k) \exp \left(\sum_{\ell=\tau(k)}^{n-1} P(\ell) \exp \left(\sum_{j=\tau(\ell)}^{\ell-1} P(j) \frac{x(\tau(j))}{x(j)}\right)\right) \leq 0 .
\end{aligned}
$$

Furthermore,

$$
\Delta x(n)=-\sum_{i=1}^{m} p_{i}(n) x\left(\tau_{i}(n)\right) \leq-x(\tau(n)) \sum_{i=1}^{m} p_{i}(n),
$$

i.e.,

$$
\Delta x(n) \leq-P(n) x(\tau(n)) .
$$

Combining (2.11) and (2.12), we have

$$
\begin{aligned}
& \Delta x(n)+P(n) x(n) \\
& +P(n) x(n) \sum_{k=\tau(n)}^{n-1} P(k) \exp \left(\sum_{\ell=\tau(k)}^{n-1} P(\ell) \exp \left(\sum_{j=\tau(\ell)}^{\ell-1} P(j) \frac{x(\tau(j))}{x(j)}\right)\right) \leq 0 .
\end{aligned}
$$

Since $\tau(j) \leq h(j)$, clearly

$$
\begin{aligned}
& \Delta x(n)+P(n) x(n) \\
& +P(n) x(n) \sum_{k=\tau(n)}^{n-1} P(k) \exp \left(\sum_{\ell=\tau(k)}^{n-1} P(\ell) \exp \left(\sum_{j=\tau(\ell)}^{\ell-1} P(j) \frac{x(h(j))}{x(j)}\right)\right) \leq 0 .
\end{aligned}
$$

Taking into account the fact that (2.2) of Lemma 2.2 is satisfied, the last inequality becomes

$$
\begin{aligned}
\Delta x(n) & +P(n) x(n) \\
& +P(n) x(n) \sum_{k=\tau(n)}^{n-1} P(k) \exp \left(\sum_{\ell=\tau(k)}^{n-1} P(\ell) \exp \left(\left(\lambda_{0}-\epsilon\right) \sum_{j=\tau(\ell)}^{\ell-1} P(j)\right)\right) \leq 0 .
\end{aligned}
$$


Thus

$$
\begin{aligned}
& \Delta x(n) \\
& +P(n)\left[1+\sum_{k=\tau(n)}^{n-1} P(k) \exp \left(\sum_{\ell=\tau(k)}^{n-1} P(\ell) \exp \left(\left(\lambda_{0}-\epsilon\right) \sum_{j=\tau(\ell)}^{\ell-1} P(j)\right)\right] x(n) \leq 0,\right.
\end{aligned}
$$

or

$$
\Delta x(n)+D_{0}(n, \epsilon) x(n) \leq 0,
$$

with

$$
D_{0}(n, \epsilon)=P(n)\left[1+\sum_{k=\tau(n)}^{n-1} P(k) \exp \left(\sum_{\ell=\tau(k)}^{n-1} P(\ell) \exp \left(\left(\lambda_{0}-\epsilon\right) \sum_{j=\tau(\ell)}^{\ell-1} P(j)\right)\right] .\right.
$$

Applying the discrete Grönwall inequality in (2.13), we obtain

$$
x(m)>x(n) \prod_{u=m}^{n-1} \frac{1}{1-D_{0}(u, \epsilon)}, \quad n \geq m .
$$

Thus

$$
x(\tau(j))>x(j) \prod_{u=\tau(j)}^{j-1} \frac{1}{1-D_{0}(u, \epsilon)}, \quad \text { for all } n \geq n(\epsilon) .
$$

Combining the inequalities (2.9) and (2.14) we obtain

$$
x(\tau(k)) \geq x(n) \exp \left(\sum_{\ell=\tau(k)}^{n-1} P(\ell) \exp \left(\sum_{j=\tau(\ell)}^{\ell-1} P(j) \prod_{u=\tau(j)}^{j-1} \frac{1}{1-D_{0}(u, \epsilon)}\right)\right) .
$$

In view of this, (2.10) becomes

$$
\begin{aligned}
& x(n)-x(\tau(n))+x(n) \times \\
& \times \sum_{k=\tau(n)}^{n-1} P(k) \exp \left(\sum_{\ell=\tau(k)}^{n-1} P(\ell) \exp \left(\sum_{j=\tau(\ell)}^{\ell-1} P(j) \prod_{u=\tau(j)}^{j-1} \frac{1}{1-D_{0}(u, \epsilon)}\right)\right) \leq 0 .
\end{aligned}
$$

Multiplying the last inequality by $P(n)$, we get

$$
\begin{aligned}
& P(n) x(n)-P(n) x(\tau(n))+P(n) x(n) \times \\
& \times \sum_{k=\tau(n)}^{n-1} P(k) \exp \left(\sum_{\ell=\tau(k)}^{n-1} P(\ell) \exp \left(\sum_{j=\tau(\ell)}^{\ell-1} P(j) \prod_{u=\tau(j)}^{j-1} \frac{1}{1-D_{0}(u, \epsilon)}\right)\right) \leq 0 .
\end{aligned}
$$


which, in view of (2.12), becomes

$$
\begin{aligned}
& \Delta x(n)+P(n) x(n)+P(n) x(n) \times \\
& \times \sum_{k=\tau(n)}^{n-1} P(k) \exp \left(\sum_{\ell=\tau(k)}^{n-1} P(\ell) \exp \left(\sum_{j=\tau(\ell)}^{\ell-1} P(j) \prod_{u=\tau(j)}^{j-1} \frac{1}{1-D_{0}(u, \epsilon)}\right)\right) \leq 0 .
\end{aligned}
$$

or

$$
\begin{aligned}
& \Delta x(n)+P(n) x(n) \times \\
& \times\left[1+\sum_{k=\tau(n)}^{n-1} P(k) \exp \left(\sum_{\ell=\tau(k)}^{n-1} P(\ell) \exp \left(\sum_{j=\tau(\ell)}^{\ell-1} P(j) \prod_{u=\tau(j)}^{j-1} \frac{1}{1-D_{0}(u, \epsilon)}\right)\right] \leq 0 .\right.
\end{aligned}
$$

Hence, for sufficiently large $n$

$$
\Delta x(n)+D_{1}(n, \epsilon) x(n) \leq 0
$$

where

$$
\begin{aligned}
& D_{1}(n, \epsilon)=P(n) \times \\
& \times\left[1+\sum_{k=\tau(n)}^{n-1} P(k) \exp \left(\sum_{\ell=\tau(k)}^{n-1} P(\ell) \exp \left(\sum_{j=\tau(\ell)}^{\ell-1} P(j) \prod_{u=\tau(j)}^{j-1} \frac{1}{1-D_{0}(u, \epsilon)}\right)\right)\right] .
\end{aligned}
$$

By induction, we can build inequalities on $\Delta x(n)$ progressively higher indices $D_{\xi}(n, \epsilon)$, $\xi \in \mathbb{N}$. In general, for sufficiently large $n$, the positive solution $x(n)$ satisfies the inequality

$$
\Delta x(n)+D_{\xi}(n, \epsilon) x(n) \leq 0
$$

where

$$
\begin{aligned}
& D_{\xi}(n, \epsilon)=P(n) \times \\
& \times\left[1+\sum_{k=\tau(n)}^{n-1} P(k) \exp \left(\sum_{\ell=\tau(k)}^{n-1} P(\ell) \exp \left(\sum_{j=\tau(\ell)}^{\ell-1} P(j) \prod_{u=\tau(j)}^{j-1} \frac{1}{1-D_{\xi-1}(u, \epsilon)}\right)\right]\right.
\end{aligned}
$$

and

$$
\begin{aligned}
x(\tau(k)) & \geq x(h(n)) \times \\
& \times \exp \left(\sum_{\ell=\tau(k)}^{h(n)-1} P(\ell) \exp \left(\sum_{j=\tau(\ell)}^{\ell-1} P(j) \prod_{u=\tau(j)}^{j-1} \frac{1}{1-D_{\xi}(u, \epsilon)}\right)\right) .
\end{aligned}
$$


Summing up (E) from $h(n)$ to $n$ and using (2.17), we have, for all sufficiently large $n$,

$$
\begin{aligned}
& x(n+1)-x(h(n))+x(h(n)) \times \\
& \times \sum_{k=h(n)}^{n} P(k) \exp \left(\sum_{\ell=\tau(k)}^{h(n)-1} P(\ell) \exp \left(\sum_{j=\tau(\ell)}^{\ell-1} P(j) \prod_{u=\tau(j)}^{j-1} \frac{1}{1-D_{\xi}(u, \epsilon)}\right)\right) \leq 0 .
\end{aligned}
$$

The inequality is valid if we omit $x(n+1)>0$ in the left-hand side.Thus, as $x(h(n))>$ 0 , for all sufficiently large $n$ it holds

$$
\sum_{k=h(n)}^{n} P(k) \exp \left(\sum_{\ell=\tau(k)}^{h(n)-1} P(\ell) \exp \left(\sum_{j=\tau(\ell)}^{\ell-1} P(j) \prod_{u=\tau(j)}^{j-1} \frac{1}{1-D_{\xi}(u, \epsilon)}\right)\right)<1,
$$

from which by letting $n \rightarrow \infty$, we have

$$
\limsup _{n \rightarrow \infty} \sum_{k=h(n)}^{n} P(k) \exp \left(\sum_{\ell=\tau(k)}^{h(n)-1} P(\ell) \exp \left(\sum_{j=\tau(\ell)}^{\ell-1} P(j) \prod_{u=\tau(j)}^{j-1} \frac{1}{1-D_{\xi}(u, \epsilon)}\right)\right) \leq 1 .
$$

Since $\epsilon$ may be taken arbitrarily small, this inequality contradicts (2.4).

The proof of the theorem is complete.

Theorem 2.5. Assume that $h(n)$ is defined by (1.4). If for some $\xi \in \mathbb{N}$

$$
\begin{aligned}
\limsup _{n \rightarrow \infty} & \sum_{k=h(n)}^{n} P(k) \exp \left(\sum_{\ell=\tau(k)}^{h(n)-1} P(\ell) \exp \left(\sum_{j=\tau(\ell)}^{\ell-1} P(j) \prod_{u=\tau(j)}^{j-1} \frac{1}{1-D_{\xi}(u)}\right)\right) \\
>1-D(\alpha), &
\end{aligned}
$$

where $D_{\xi}(n)$ is defined by (2.5), then all solutions of $(E)$ are oscillatory.

Proof. Assume, for the sake of contradiction, that $(x(n))_{n \geq-v}$ is a nonoscillatory solution of $(E)$. Since $(-x(n))_{n>-v}$ is also a solution of $(E)$, we can confine our discussion only to the case where $x(n)>0$, for all large $n$. Then, as in the proof of Theorem 2.4, for sufficiently large $n,(2.18)$ is satisfied, i.e.,

$$
\begin{aligned}
& x(n+1)-x(h(n))+x(h(n)) \times \\
& \times \sum_{k=h(n)}^{n} P(k) \exp \left(\sum_{\ell=\tau(k)}^{h(n)-1} P(\ell) \exp \left(\sum_{j=\tau(\ell)}^{\ell-1} P(j) \prod_{u=\tau(j)}^{j-1} \frac{1}{1-D_{\xi}(u, \epsilon)}\right)\right) \leq 0 .
\end{aligned}
$$


That is,

$$
\begin{aligned}
& \sum_{k=h(n)}^{n} P(k) \exp \left(\sum_{\ell=\tau(k)}^{h(n)-1} P(\ell) \exp \left(\sum_{j=\tau(\ell)}^{\ell-1} P(j) \prod_{u=\tau(j)}^{j-1} \frac{1}{1-D_{\xi}(u, \epsilon)}\right)\right) \\
& \leq 1-\frac{x(n+1)}{x(h(n))},
\end{aligned}
$$

which gives

$$
\begin{aligned}
& \limsup _{n \rightarrow \infty} \sum_{k=h(n)}^{n} P(k) \exp \left(\sum_{\ell=\tau(k)}^{h(n)-1} P(\ell) \exp \left(\sum_{j=\tau(\ell)}^{\ell-1} P(j) \prod_{u=\tau(j)}^{j-1} \frac{1}{1-D_{\xi}(u, \epsilon)}\right)\right) \\
& \leq 1-\liminf _{n \rightarrow \infty} \frac{x(n+1)}{x(h(n))} .
\end{aligned}
$$

Since $0<\alpha \leq 1 / e$, by Lemma 2.3, inequality (2.3) holds. So the last inequality leads to

$$
\begin{aligned}
& \limsup _{n \rightarrow \infty} \sum_{k=h(n)}^{n} P(k) \exp \left(\sum_{\ell=\tau(k)}^{h(n)-1} P(\ell) \exp \left(\sum_{j=\tau(\ell)}^{\ell-1} P(j) \prod_{u=\tau(j)}^{j-1} \frac{1}{1-D_{\xi}(u, \epsilon)}\right)\right) \\
& \leq 1-D(\alpha) .
\end{aligned}
$$

Since $\epsilon$ may be taken arbitrarily small, this inequality contradicts (2.19).

The proof of the theorem is complete.

Theorem 2.6. Assume that $h(n)$ is defined by (1.4). If for some $\xi \in \mathbb{N}$

$$
\begin{aligned}
& \limsup _{n \rightarrow \infty} \sum_{k=h(n)}^{n} P(k) \exp \left(\sum_{\ell=\tau(k)}^{n} P(\ell) \exp \left(\sum_{j=\tau(\ell)}^{\ell-1} P(j) \prod_{u=\tau(j)}^{j-1} \frac{1}{1-D_{\xi}(u)}\right)\right) \\
& >\frac{1}{D(\alpha)}-1,
\end{aligned}
$$

where $D_{\xi}(n)$ by (2.5), then all solutions of $(E)$ are oscillatory.

Proof. Assume, for the sake of contradiction, that $(x(n))_{n \geq-v}$ is an eventually positive solution of $(E)$. Then, as in the proof of Theorem 2.4, (2.17) is satisfied, i.e.,

$$
x(\tau(k)) \geq x(h(n)) \exp \left(\sum_{\ell=\tau(k)}^{h(n)-1} P(\ell) \exp \left(\sum_{j=\tau(\ell)}^{\ell-1} P(j) \prod_{u=\tau(j)}^{j-1} \frac{1}{1-D_{\xi}(u, \epsilon)}\right)\right) .
$$


Therefore, for a sufficiently large $n$, we have

$$
\begin{aligned}
& x(\tau(k)) \\
& \geq x(n+1) \exp \left(\sum_{\ell=\tau(k)}^{n} P(\ell) \exp \left(\sum_{j=\tau(\ell)}^{\ell-1} P(j) \prod_{u=\tau(j)}^{j-1} \frac{1}{1-D_{\xi}(u, \epsilon)}\right)\right) .
\end{aligned}
$$

Summing up (E) from $h(n)$ to $n$, we have

$$
x(n+1)-x(h(n))+\sum_{k=h(n)}^{n} \sum_{i=1}^{m} p_{i}(k) x\left(\tau_{i}(k)\right)=0,
$$

or

$$
x(n+1)-x(h(n))+\sum_{k=h(n)}^{n}\left(\sum_{i=1}^{m} p_{i}(k)\right) x(\tau(k)) \leq 0,
$$

i.e.,

$$
x(n+1)-x(h(n))+\sum_{k=h(n)}^{n} P(k) x(\tau(k)) \leq 0 .
$$

In view of (2.21), the last inequality gives

$$
\begin{aligned}
& x(n+1)-x(h(n))+\sum_{k=h(n)}^{n} P(k) x(n+1) \times \\
& \times \exp \left(\sum_{\ell=\tau(k)}^{n} P(\ell) \exp \left(\sum_{j=\tau(\ell)}^{\ell-1} P(j) \prod_{u=\tau(j)}^{j-1} \frac{1}{1-D_{\xi}(u, \epsilon)}\right)\right) \leq 0,
\end{aligned}
$$

or

$$
\begin{aligned}
& x(n+1)-x(h(n))+x(h(n)) \times \\
& \times \sum_{k=h(n)}^{n} P(k) \frac{x(n+1)}{x(h(n))} \times \\
& \times \exp \left(\sum_{\ell=\tau(k)}^{n} P(\ell) \exp \left(\sum_{j=\tau(\ell)}^{\ell-1} P(j) \prod_{u=\tau(j)}^{j-1} \frac{1}{1-D_{\xi}(u, \epsilon)}\right)\right) \leq 0,
\end{aligned}
$$

or

$$
\begin{aligned}
& x(n+1)+x(h(n)) \times\left[\frac{x(n+1)}{x(h(n))} \sum_{k=h(n)}^{n} P(k) \times\right. \\
& \left.\times \exp \left(\sum_{\ell=\tau(k)}^{n} P(\ell) \exp \left(\sum_{j=\tau(\ell)}^{\ell-1} P(j) \prod_{u=\tau(j)}^{j-1} \frac{1}{1-D_{\xi}(u, \epsilon)}\right)\right)-1\right] \leq 0 .
\end{aligned}
$$


Thus, for all sufficiently large $n$, it holds

$$
\begin{aligned}
& \sum_{k=h(n)}^{n} P(k) \exp \left(\sum_{\ell=\tau(k)}^{n} P(\ell) \exp \left(\sum_{j=\tau(\ell)}^{\ell-1} P(j) \prod_{u=\tau(j)}^{j-1} \frac{1}{1-D_{\xi}(u, \epsilon)}\right)\right) \\
& \leq \frac{x(h(n))}{x(n+1)}-1 .
\end{aligned}
$$

Letting $n \rightarrow \infty$, we take

$$
\begin{aligned}
& \limsup _{n \rightarrow \infty} \sum_{k=h(n)}^{n} P(k) \exp \left(\sum_{\ell=\tau(k)}^{n} P(\ell) \exp \left(\sum_{j=\tau(\ell)}^{\ell-1} P(j) \prod_{u=\tau(j)}^{j-1} \frac{1}{1-D_{\xi}(u, \epsilon)}\right)\right) \\
& \leq \limsup _{n \rightarrow \infty} \frac{x(h(n))}{x(n+1)}-1 .
\end{aligned}
$$

Since $0<\alpha \leq 1 / e$, by Lemma 2.3, inequality (2.3) holds. So the last inequality leads to

$$
\begin{aligned}
& \limsup _{n \rightarrow \infty} \sum_{k=h(n)}^{n} P(k) \exp \left(\sum_{\ell=\tau(k)}^{n} P(\ell) \exp \left(\sum_{j=\tau(\ell)}^{\ell-1} P(j) \prod_{u=\tau(j)}^{j-1} \frac{1}{1-D_{\xi}(u, \epsilon)}\right)\right) \\
& \leq \frac{1}{D(\alpha)}-1 .
\end{aligned}
$$

Since $\epsilon$ may be taken arbitrarily small, this inequality contradicts (2.20).

The proof of the theorem is complete.

Remark 2.7. If $D_{\xi}(n, \epsilon) \geq 1$, then (2.16) guarantees that all solutions of ( $E$ ) are oscillatory. In fact, (2.16) gives

$$
\Delta x(n)+x(n) \leq 0
$$

which means that $x(n+1) \leq 0$. This contradicts $x(n)>0$, for all $n \geq n_{2}$. Thus, in Theorems 2.4, 2.5 and 2.6 we consider only the case $D_{\xi}(n)<1$. Another conclusion, that can be drawn from the above, is that if at some point through the iterative process, we get a value of $\xi$, for which $D_{\xi}(n) \geq 1$, then the process terminates, since in any case, all solutions of $(E)$ will be oscillatory. The value of $\xi$, that is the number of iterations, obviously, depends on the coefficients $p_{i}(n)$ and the form of the nonmonotone arguments $\tau_{i}(n)$.

Example 2.8. Consider the retarded difference equation

$$
\Delta x(n)+\frac{717}{10000} x\left(\tau_{1}(n)\right)+\frac{239}{10000} x\left(\tau_{2}(n)\right)=0, \quad n \in \mathbb{N}_{0},
$$


with (see Fig. 2.1, (a))

$$
\tau_{1}(n)=\left\{\begin{array}{ll}
n-1, & \text { if } n=5 \mu \\
n-6, & \text { if } n=5 \mu+1 \\
n-2, & \text { if } n=5 \mu+2 \\
n-6, & \text { if } n=5 \mu+3 \\
n-3, & \text { if } n=5 \mu+4
\end{array} \quad \text { and } \quad \tau_{2}(n)=\tau_{1}(n)-1\right.
$$

where $\mu \in \mathbb{N}_{0}$ and $\mathbb{N}_{0}$ is the set of nonnegative integers. By (1.4), we see (Fig. 2.1, (b))

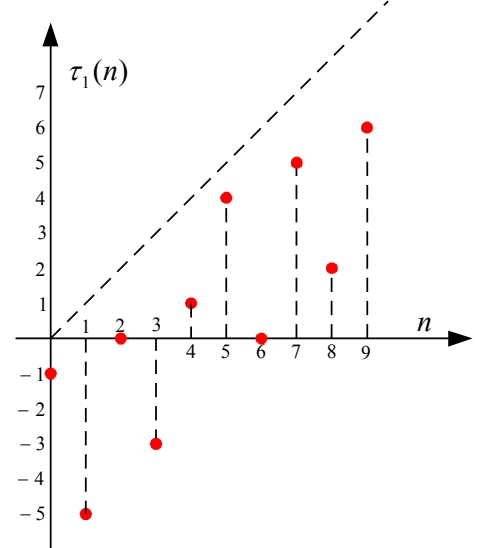

(a)

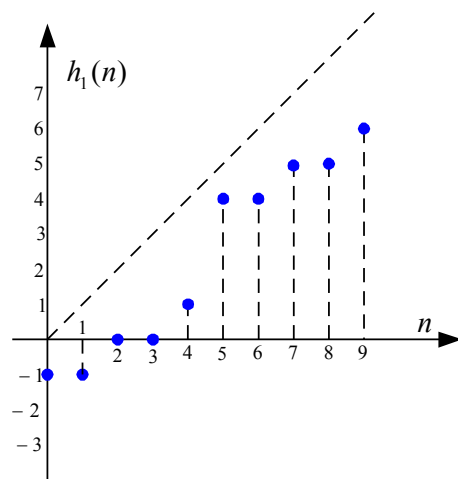

(b)

Figure 2.1: The graphs of $\tau_{1}(n)$ and $h_{1}(n)$

that

$$
h_{1}(n)=\left\{\begin{array}{ll}
n-1, & \text { if } n=5 \mu \\
n-2, & \text { if } n=5 \mu+1 \\
n-2, & \text { if } n=5 \mu+2 \\
n-3, & \text { if } n=5 \mu+3 \\
n-3, & \text { if } n=5 \mu+4
\end{array} \quad \text { and } \quad h_{2}(n)=h_{1}(n)-1\right.
$$

Consequently,

$$
h(n)=\max _{1 \leq i \leq 2}\left\{h_{i}(n)\right\}=h_{1}(n) \text { and } \tau(n)=\max _{1 \leq i \leq 2} \tau_{i}(n)=\tau_{1}(n) .
$$

It is easy to see that

$$
\begin{aligned}
\alpha & =\liminf _{n \rightarrow \infty} \sum_{i=1}^{2} \sum_{j=\tau(n)}^{n-1} p_{i}(j) \\
& =\liminf _{\mu \rightarrow \infty} \sum_{i=1}^{2} \sum_{j=5 \mu-1}^{5 \mu-1} p_{i}(j)=\frac{717}{10000}+\frac{239}{10000}=0.0956
\end{aligned}
$$


and the smaller root of equation $e^{0.0956 \lambda}=\lambda$ is $\lambda_{0} \simeq 1.11218$.

Clearly, $\sum_{i=1}^{2} p_{i}(n)=0.0956<1$ and therefore (1.5) is satisfied for all $n \geq 0$.

Observe that the function $F_{\xi}: \mathbb{N}_{0} \rightarrow \mathbb{R}_{+}$defined as

$$
F_{\xi}(n)=\sum_{k=h(n)}^{n} P(k) \exp \left(\sum_{\ell=\tau(k)}^{h(n)-1} P(\ell) \exp \left(\sum_{j=\tau(\ell)}^{\ell-1} P(j) \prod_{u=\tau(j)}^{j-1} \frac{1}{1-D_{\xi}(u)}\right)\right)
$$

attains its maximum at $n=5 \mu+4, \mu \in \mathbb{N}_{0}$, for every $\xi \in \mathbb{N}$. Specifically, by using an algorithm on MATLAB software, we obtain

$$
\begin{aligned}
F_{1}(5 \mu+4) & =\sum_{k=5 \mu+1}^{5 \mu+4} P(k) \exp \left(\sum_{\ell=\tau(k)}^{5 \mu} P(\ell) \exp \left(\sum_{j=\tau(\ell)}^{\ell-1} P(j) \prod_{u=\tau(j)}^{j-1} \frac{1}{1-D_{1}(u)}\right)\right) \\
& \simeq 1.015
\end{aligned}
$$

and therefore

$$
\limsup _{n \rightarrow \infty} F_{1}(n) \simeq 1.015>1 \text {. }
$$

That is, condition (2.4) of Theorem 2.4 is satisfied, for $\xi=1$, which means that all solutions of (2.22) are oscillatory.

Observe, however, that

$$
\alpha=0.0956<\frac{1}{e}
$$

and

$$
\limsup _{n \rightarrow \infty} \sum_{i=1}^{m} \sum_{j=\tau(n)}^{n} p_{i}(j)=\limsup _{\mu \rightarrow \infty} \sum_{i=1}^{2} \sum_{j=5 \mu+1}^{5 \mu+4} p_{i}(j)=4 \cdot 0.0956=0.3824<1 .
$$

Also,

$$
\begin{aligned}
& \sum_{j=5 \mu+1}^{5 \mu+4} \sum_{i=1}^{2} p_{i}(j) a_{1}^{-1}\left(5 \mu+1, \tau_{i}(j)\right) \\
= & \frac{717}{10000} a_{1}^{-1}(5 \mu+1,5 \mu-5)+\frac{239}{10000} a_{1}^{-1}(5 \mu+1,5 \mu-6) \\
& +\frac{717}{10000} a_{1}^{-1}(5 \mu+1,5 \mu)+\frac{239}{10000} a_{1}^{-1}(5 \mu+1,5 \mu-1) \\
& +\frac{717}{10000} a_{1}^{-1}(5 \mu+1,5 \mu-3)+\frac{239}{10000} a_{1}^{-1}(5 \mu+1,5 \mu-4) \\
& +\frac{717}{10000} a_{1}^{-1}(5 \mu+1,5 \mu+1)+\frac{239}{10000} a_{1}^{-1}(5 \mu+1,5 \mu) \\
= & \frac{717}{10000} \frac{1}{(1-0.0956)^{6}}+\frac{239}{10000} \frac{1}{(1-0.0956)^{7}}
\end{aligned}
$$




$$
\begin{aligned}
& +\frac{717}{10000} \frac{1}{1-0.0956}+\frac{239}{10000} \frac{1}{(1-0.0956)^{2}} \\
& +\frac{717}{10000} \frac{1}{(1-0.0956)^{4}}+\frac{239}{10000} \frac{1}{(1-0.0956)^{5}} \\
& +\frac{717}{10000} \cdot 1+\frac{239}{10000} \frac{1}{1-0.0956} \simeq 0.5326 .
\end{aligned}
$$

Thus,

$$
\limsup _{n \rightarrow \infty} \sum_{j=h(n)}^{n} \sum_{i=1}^{3} p_{i}(j) a_{1}^{-1}\left(h(n), \tau_{i}(j)\right) \simeq 0.5326<1 .
$$

Finally, by using algorithms on MATLAB software, we obtain

$$
\begin{gathered}
\limsup _{n \rightarrow \infty} \sum_{j=h(n)}^{n} \mathcal{P}(j) \prod_{i=\tau(j)}^{h(n)-1} \frac{1}{1-\mathcal{P}_{1}(i)} \simeq 0.6116<1, \\
0.6116<1-D(\alpha) \simeq 0.9949, \\
\limsup _{n \rightarrow \infty} \sum_{\ell=h(n)}^{n} P(\ell) \exp \left(\sum_{j=\tau(\ell)}^{h(n)-1} P(j) \prod_{u=\tau(j)}^{j-1} \frac{1}{1-Z_{1}(u)}\right) \simeq 0.8294<1, \\
0.8294<1-D(\alpha) \simeq 0.9949 .
\end{gathered}
$$

That is, none of conditions (1.2), (1.3), (1.6) (for $r=1$ ), (1.7) (for $\ell=1$ ), (1.8) (for $\ell=1$ ), (1.9) (for $w=1$ ), and (1.10) (for $w=1$ ) is satisfied.

Comments. It is worth noting that condition (2.4) achieves a significant improvement, approximately $165.43 \%$, over the corresponding condition (1.3). We get that estimate by comparing the values on the left-side of these conditions. The improvement over the conditions (1.6), (1.7) and (1.9) is very satisfactory, around $90.57 \%, 65.96 \%$ and $22.38 \%$, respectively.

Finally, observe that conditions (1.6), (1.7), (1.8), (1.9) and (1.10) do not lead to oscillation, from the first iteration. On the contrary, condition (2.4) is satisfied from the first iteration. This means that our condition is better and much faster than (1.6), (1.7), (1.8), (1.9) and (1.10).

Remark 2.9. Similarly, one can construct examples to illustrate the other main results.

\section{Assessment of Main Results and Concluding Remarks}

In the present paper, we have considered the oscillatory dynamics of retarded difference equations having nonmonotone arguments and nonnegative coefficients. New sufficient 
conditions for the oscillation of all solutions of $(E)$ have been established, which include (2.4), (2.19) and (2.20). Applying these conditions involves a procedure that checks for oscillations by iteratively computing limsup on terms recursively defined on the equation's coefficients and retarded arguments.

The main advantage of these conditions is that they achieve a major improvement over all the related oscillation conditions for $(E)$ in the literature. For example, condition (2.4) improves upon the non-iterative condition that is reviewed in the introduction, namely condition (1.3). That becomes evident immediately by inspecting the left-hand side of (2.4) and the left-hand side of (1.3). Indeed, since

$$
\exp \left(\sum_{\ell=\tau(k)}^{h(n)-1} P(\ell) \exp \left(\sum_{j=\tau(\ell)}^{\ell-1} P(j) \prod_{u=\tau(j)}^{j-1} \frac{1}{1-D_{\xi}(u)}\right)\right) \geq 1,
$$

it is clear that

$$
\begin{aligned}
& \sum_{k=h(n)}^{n} P(k) \exp \left(\sum_{\ell=\tau(k)}^{h(n)-1} P(\ell) \exp \left(\sum_{j=\tau(\ell)}^{\ell-1} P(j) \prod_{u=\tau(j)}^{j-1} \frac{1}{1-D_{\xi}(u)}\right)\right) \\
& \geq \sum_{i=1}^{m} \sum_{j=h(n)}^{n} p_{i}(j) .
\end{aligned}
$$

This means that the condition (2.4) is weaker than (1.3).

Also, since

$$
D_{0}(n) \geq Z_{0}(n)>\mathcal{P}_{0}(n)=P(n),
$$

clearly

$$
\begin{aligned}
& \sum_{k=h(n)}^{n} P(k) \exp \left(\sum_{\ell=\tau(k)}^{h(n)-1} P(\ell) \exp \left(\sum_{j=\tau(\ell)}^{\ell-1} P(j) \prod_{u=\tau(j)}^{j-1} \frac{1}{1-D_{\xi}(u)}\right)\right) \\
\geq & \sum_{\ell=h(n)}^{n} P(\ell) \exp \left(\sum_{j=\tau(\ell)}^{h(n)-1} P(j) \prod_{u=\tau(j)}^{j-1} \frac{1}{1-Z_{w}(u)}\right) \\
\geq & \sum_{j=h(n)}^{n} P(j) \prod_{i=\tau(j)}^{h(n)-1} \frac{1}{1-\mathcal{P}_{\ell}(i)} .
\end{aligned}
$$

Therefore, the condition (2.4) is weaker than conditions (1.8) and (1.9).

The improvement of (2.4) over the other iterative conditions, namely (1.6) (for $r>$ 1), (1.7) (for $\ell>1$ ), (1.8) (for $\ell>1$ ), (1.9) (for $w>1$ ), (1.10) (for $w>1$ )] and (1.11) (for $w>1$ ), is that it requires far fewer iterations to establish oscillation than the other conditions. 
This advantage can be easily verified computationally by running the MATLAB programs for computing lim sup and comparing the number of iterations required by each condition to establish oscillation. Then we see that we achieve a significant improvement over all known oscillation criteria.

\section{References}

[1] L. Berezansky and E. Braverman, On existence of positive solutions for linear difference equations with several delays, Advances Dyn. Syst. Appl., 1 (2006), 29_ 47.

[2] E. Braverman, G. E. Chatzarakis and I. P. Stavroulakis, Iterative oscillation tests for difference equations with several nonmonotone arguments, J. Difference Equ. Appl., 21 (2015), No. 9, 854-874.

[3] G. E. Chatzarakis, L. Horvat-Dmitrović and M. Pašić, Oscillation tests for Difference Equations with several nonmonotone deviating arguments, Math. Slovaca, 68 (2018), No. 5, 1083-1096.

[4] G. E. Chatzarakis and I. Jadlovská, Oscillations in difference equations with several arguments using an iterative method, Filomat, 32 (2018), No. 1, 255-273.

[5] G. E. Chatzarakis and I. Jadlovská, Difference equations with several nonmonotone deviating arguments: Iterative oscillation tests, Dynamic Systems and Applications, 27 (2018), No. 2, 271-298.

[6] G. E. Chatzarakis and I. Jadlovská, Iterative oscillation criteria in deviating difference equations, Mediterr. J. Math. 16(2019), no. 1, 1-16.

[7] G. E. Chatzarakis, T. Kusano and I. P. Stavroulakis, Oscillation conditions for difference equations with several variable arguments, Math. Bohem., 140 (2015), No. 3, 291-311.

[8] G. E. Chatzarakis, J. Manojlovic, S. Pinelas and I. P. Stavroulakis, Oscillation criteria of difference equations with several deviating arguments, Yokohama Math. J., 60 (2014), 13-31.

[9] G. E. Chatzarakis and M. Pašić, Improved iterative oscillation tests in difference equations with several arguments, J. Difference Equ. Appl., 25 (2019), no. 1, 64 83.

[10] G. E. Chatzarakis, S. Pinelas and I. P. Stavroulakis, Oscillations of difference equations with several deviated arguments, Aequat. Math., 88 (2014), 105-123. 
[11] L.H. Erbe, Q.K. Kong and B.G. Zhang, Oscillation Theory for Functional Differential Equations, Marcel Dekker, New York, 1995.

[12] X. Li and D. Zhu, Oscillation of advanced difference equations with variable coefficients, Ann. Differential Equations, 18 (2002), 254-263.

[13] X. N. Luo, Y. Zhou and C. F. Li, Oscillations of a nonlinear difference equation with several delays, Mathematica Bohemica, 128 (2003), 309-317.

[14] X. H. Tang and J. S. Yu, Oscillation of delay difference equations, Comput. Math. Appl., 37 (1999), 11-20.

[15] X. H. Tang and R. Y. Zhang, New oscillation criteria for delay difference equations, Comput. Math. Appl., 42 (2001), 1319-1330.

[16] X. Wang, Oscillation of delay difference equations with several delays, J. Math. Anal. Appl., 286 (2003), 664-674.

[17] X. P. Wu and L. Wang, Zero-Hopf bifurcation analysis in delayed differential equations with two delays, J. Frankl. Inst., 354 (2017), 1484-1513.

[18] W. Yan, Q. Meng and J. Yan, Oscillation criteria for difference equation of variable delays, DCDIS Proceedings 3 (2005), 641-647.

[19] B. G. Zhang and C. J. Tian, Nonexistence and existence of positive solutions for difference equations with unbounded delay, Comput. Math. Appl., 36 (1998), 1-8.

[20] B. G. Zhang and Y. Zhou, Oscillations of difference equations with several delays, Comput. Math. Appl., 44 (2002), 817-821. 